\title{
Comparison between the different methods developed for determining the onset of the LH surge in urine during the human menstrual cycle
}

\author{
Sonya Godbert ${ }^{1} \cdot$ Fernando Miro $^{1} \cdot$ Chris Shreeves $^{1} \cdot$ Christian Gnoth $^{2,3} \cdot$ \\ Sarah Johnson ${ }^{1}$
}

Received: 24 February 2015/ Accepted: 20 April 2015/Published online: 5 May 2015

(C) The Author(s) 2015. This article is published with open access at Springerlink.com

\begin{abstract}
Purpose To determine whether an optimal method exists for the detection of the luteinising hormone (LH) surge onset in research datasets of urinary hormonal profiles of menstrual cycles.

Methods The scientific literature was searched to compare published methodologies for detection of the LH surge onset in urine. Their performance was tested using complete hormonal profiles from 254 ovulatory cycles from 227 women attempting pregnancy (normal regular menstrual cycles; no known infertility).

Results Three major methodologies to determine the onset of the LH surge in urine were identified. The key difference between these methods is how the cycle days that contribute to LH baseline assessment are determined: using fixed days (method \#1), based on peak LH day (method \#2), based on a provisional estimate of the LH surge (method \#3). Method \#1 requires no prior cycle information, whereas methods \#2 and \#3 need to consider complete cycle data. The most reliable method for calculation of baseline LH was using 2 days before the estimated surge day, plus the previous $4 / 5$ days.

Conclusions Different methods for identification of the urinary LH surge can provide very different determinations of LH surge day, thus care must be taken when comparing
\end{abstract}

Sarah Johnson

sarah.johnson@spdspark.com

1 SPD Development Company Ltd, Priory Business Park, Stannard Way, Bedford MK44 3UP, UK

2 green-ivf, Grevenbroich Endocrinology and IVF-Center, Rheydter Str. 143, 41515 Grevenbroich, Germany

3 Department of Gynecology and Obstetrics, University of Cologne, Cologne, Germany between studies that apply different methodologies. The optimal method for determining the onset of the LH surge in urine requires retrospective estimation of day of $\mathrm{LH}$ surge to identify the most appropriate part of the cycle to consider as the baseline. This method can be adopted for application in population studies.

Keywords Luteinising hormone $\cdot$ LH $\cdot$ LH surge · Menstrual cycle

\section{Introduction}

Luteinising hormone (LH) plays a complex role in human ovarian physiology. During the follicular phase, it stimulates thecal cells to produce androgens, which are converted into oestrogens by the granulosa cells. During the luteal phase, LH has a major role in maintaining the full function of the corpus luteum. However, regarding fertility control, its most significant role is to induce ovulation at mid-cycle [1]. During ovulation, LH triggers the completion of the maturational process of the oocyte to metaphase II, and its release from the follicle. Finding reliable ways to determine the moment of ovulation is a critical task in the control of human conception and contraception. It also has implications in reproductive medicine, such as timed intercourse, artificial insemination and the diagnosis of anovulation [2].

Understanding the underlying population variability with regard to ovulation is extremely important to appropriately design methodologies to prospectively predict ovulation; for example, for the purposes of timing intercourse to achieve pregnancy. The measurement of reproductive hormones in daily urine samples during the menstrual cycle, especially LH, combines an easy way to 
collect specimens, and an objective reference to determine the onset of ovulation.

The level of LH suddenly surges, then peaks mid-cycle causing the dominant follicle to rupture and release a mature ovum. Ovulation typically occurs approximately 28-48 $\mathrm{h}$ after the beginning of the LH surge [3] and approximately 0.5 days after the peak in levels of LH [4]. Determination of peak LH is simple, but as peak urinary LH levels coincide with, or occur after ovulation, calculation of the peak LH day does not provide the advanced warning of ovulation that is needed for timing of intercourse, or fertility procedures $[3,5,6]$. In addition, urinary peak $\mathrm{LH}$ day can differ according to the assay used to measure LH in urine, with those detecting beta-core LH $(\mathrm{LH}-\beta \mathrm{c})$ identifying a peak day at least 1 day after assays that do not detect LH- $\beta$ c [4]. Identification of the day of the urinary LH surge does not appear to be influenced by the assay used, and provides 24-36 h warning of impending ovulation [7-10], therefore making it an excellent parameter to measure in order to provide prospective information regarding ovulation. Therefore, it is important to have a well-validated method for assignment of the $\mathrm{LH}$ surge and to examine retrospective datasets to provide information on population variability of LH surge.

Detecting the day of the LH surge in urine, when the first sustained rise in the level of this hormone occurs, is not straightforward. The onset of the LH surge in women of reproductive age has been investigated by many researchers, who have developed different approaches for surge identification [3, 6, 7, 10-18]. Nevertheless, no comparison has been made between these different methods to date, to determine whether they are equally valid, or whether there is one method that is more effective than the others.

The aim of this study was to compare the different methods reported in published studies that have been developed to estimate the onset of the LH surge in urine during the menstrual cycle. The study sought to identify the different methods used, and subsequently compare them on a sufficiently large number of complete LH profiles from ovulatory cycles of premenopausal fertile women, to determine the most appropriate method to use for the retrospective analysis of study populations. The results of these analyses will lead to further understanding of LH profiles in women and may assist in improving the accuracy of prospective methods of predicting ovulation based on the detection of the LH surge.

\section{Methods}

\section{Selection of peer-reviewed papers}

To identify published methods used to detect the onset of the LH surge in urine during the menstrual cycle, a thorough search of the scientific literature of peer-reviewed papers in biomedical journals was completed. PubMed and Web of Science databases were searched using the following terms: LH (all spelling variations and abbreviations); surge (all equivalent terms e.g. rise, increase). All abstracts were examined and full papers obtained if the LH surge (or equivalent) was a parameter measured in the study. Citations within identified papers were also investigated to identify further relevant papers. Out of these studies, only those in human subjects describing full methodologies to detect the LH surge during the menstrual cycle were selected for inclusion in this analysis.

\section{Testing the methodology}

From the selected studies, the algorithms employed to detect the LH surge in each case were investigated, the methodology was compared, and a classification of the different methods was established. The identified methods were then compared by analysing their performance in complete urinary hormonal profiles of a reliable sample of menstrual cycles.

\section{Menstrual cycle collection}

Complete menstrual profiles (from menses to menses) of urinary levels of $\mathrm{LH}$ and pregnanediol-3-glucuronide (PdG) were used to compare the different methods designed for detecting the LH surge during the cycle. Daily urine samples (first morning void) were collected by 227 premenopausal women, aged 18-45 years, attempting to become pregnant, with regular cycles of normal duration (21-42 days), and no known fertility problem. Volunteers were required to have two natural cycles prior to study entry and could not be using any fertility medications, breast feeding or have diagnosed polycystic ovarian syndrome. The volunteers collected daily urine samples into sample pots containing sodium azide as a preservative. Volunteers were required to refrigerate samples on collection and return them to the study site, where samples were frozen at $-80{ }^{\circ} \mathrm{C}$ prior to analysis.

\section{Sample analysis}

Hormone analyses were conducted as batch analyses, ensuring complete cycles were analysed on single assay plates. Samples were brought to room temperature and mixed prior to analysis. It had previously been determined that up to five freeze-thaw cycles had no effect on analyte concentration.

Hormonal analysis of urinary LH and PdG was conducted using AutoDELFIA (Perkin Elmer, Waltham, USA) as previously described [19]. The in-house LH assay 
consists of immobilised biotinylated antibody (antibody \#2119; SPD Development Co., Ltd, Bedford, UK) that recognises the alpha LH subunit bound to streptavidin plates, and a second, europium-labelled antibody that recognises the beta subunit (antibody \#2301; SPD Development Co., Ltd, Bedford, UK), thus it is only able to measure intact LH. Assay sensitivity was $0.1 \mathrm{mIU} / \mathrm{ml}$ and inter- and intra-assay percentage coefficient of variation (CV) was less than $5 \%$; linearity was seen in dilutions up to 1 in 20 and no high-dose hook was observed when testing up to $1000 \mathrm{mIU} / \mathrm{ml}$. The PdG assay used was also an in-house competitive immunoassay based on competition between sample and europium-labelled $\mathrm{PdG}$ for binding by a high-affinity antibody (antibody \#5806; SPD Development Co., Ltd, Bedford, UK). Assay sensitivity was $0.021 \mu \mathrm{g} / \mathrm{ml}$ and intra- and inter-assay percentage $\mathrm{CV}$ was below $10 \%$ for the standards tested $(0.16,0.80,4.00$, $20.00,100.00 \mu \mathrm{g} / \mathrm{ml}$ ); linearity was seen in up to a 1 in 20 dilution of urine sample.

\section{Cycle inclusion and analysis of methods identified in the literature for determining the urinary $\mathrm{LH}$ surge}

A total of 254 reference cycles with complete hormonal profiles of $\mathrm{LH}$ and $\mathrm{PdG}$ were included in this study. Out of these cycles, 148 ended in conception. Only hormonal profiles showing these unambiguous features of ovulation were included in the study. The existence of the LH surges followed by peaks, as well as a consistent rise in the levels of $\mathrm{PdG}$, was proved visually on the plotted hormonal profiles ( $\mathrm{LH}$ and $\mathrm{PdG}$ ). The $\mathrm{LH}$ surge day was assigned following abundant visual analysis of the graphical data by the authors. This method has been shown repeatedly to be effective and reliable in the interpretation of basal body temperature charts for assigning reference point in menstrual cycles $[20,21]$. LH and $\mathrm{PdG}$ profiles corroborate the correctness of this visually assigned LH surge; rise of urinary LH surge occurs $0.81 \pm 0.89$ days prior to ovulation and the rise of urinary $\mathrm{PdG}$ from baseline has been demonstrated to occur $3.20 \pm 1.07$ days after ovulation (Roos et al., submitted), using the assay previously reported and employed [4]. This LH surge was considered the reference surge day against which modelled surges were compared for agreement. The algorithms defined by each method were evaluated in the cycles using SAS 9.3 software. LH baseline was determined for each method for each cycle, then surge assigned for each method identified in the literature. The surge was determined as the first sustained rise in $\mathrm{LH}$ above the baseline, with a sustained rise defined as a level of at least 2.5-fold of the standard deviation (SD) over the mean baseline level.
Agreement between the reference surge and the results from the modelled surges from the different literatureidentified methods were examined to the exact day.

\section{Results}

\section{Analysis of the scientific literature}

Altogether, 12 studies investigating the onset of the LH surge in the menstrual cycle, containing a well-defined, reliable, methodology for detecting the surge in urine were identified (Table 1) [3, 6, 7, 10-18]. From the methodology described in these 12 studies, 16 different methods used for detecting the LH surge were identified. Analysis of these 16 methods found that they could be separated into three major methodological categories.

All the identified methods defined the LH surge as 'the first sustained rise in the level of LH, from the first day of the cycle'. Similarly, all methods used the mean value of 4 or 5 days to estimate baseline LH values.

The definition of the magnitude of the rise in the level of LH to identify the occurrence of the surge was defined differently in the various studies. Some studies took a multiple value of the baseline level (e.g. twice or three times the baseline level), others considered it as a number of SD values over the mean baseline level.

However, the key difference between the algorithms developed is the method used to determine the baseline level of LH during the follicular phase of the cycle from where the rise occurs and, specifically, the reference points used to estimate this baseline value.

\section{Methods identified for the detection of the onset of the $\mathrm{LH}$ surge in the menstrual cycle}

Three major categories of methods, based on estimation of the baseline values of urinary LH can be distinguished:

1. Using fixed days during the cycle. The basal level of urinary LH is determined by calculating the mean LH value from day 5 (or 6) of the cycle, and up to days 8,9 or 10. Four different variations of this method have been used: mean LH levels from days 5 to 8, from days 5 to 9 , from days 6 to 9 and from days 6 to 10. This method can be applied both retrospectively and prospectively, as it does not need data of the complete menstrual cycle.

2. Using the day of the LH peak in the cycle. Urinary LH peak day of the cycle under investigation is identified, then $3,4,5$ or 6 days prior to this is taken as a reference day, and the mean $\mathrm{LH}$ value on the reference day is then calculated, plus the level on the previous 3 


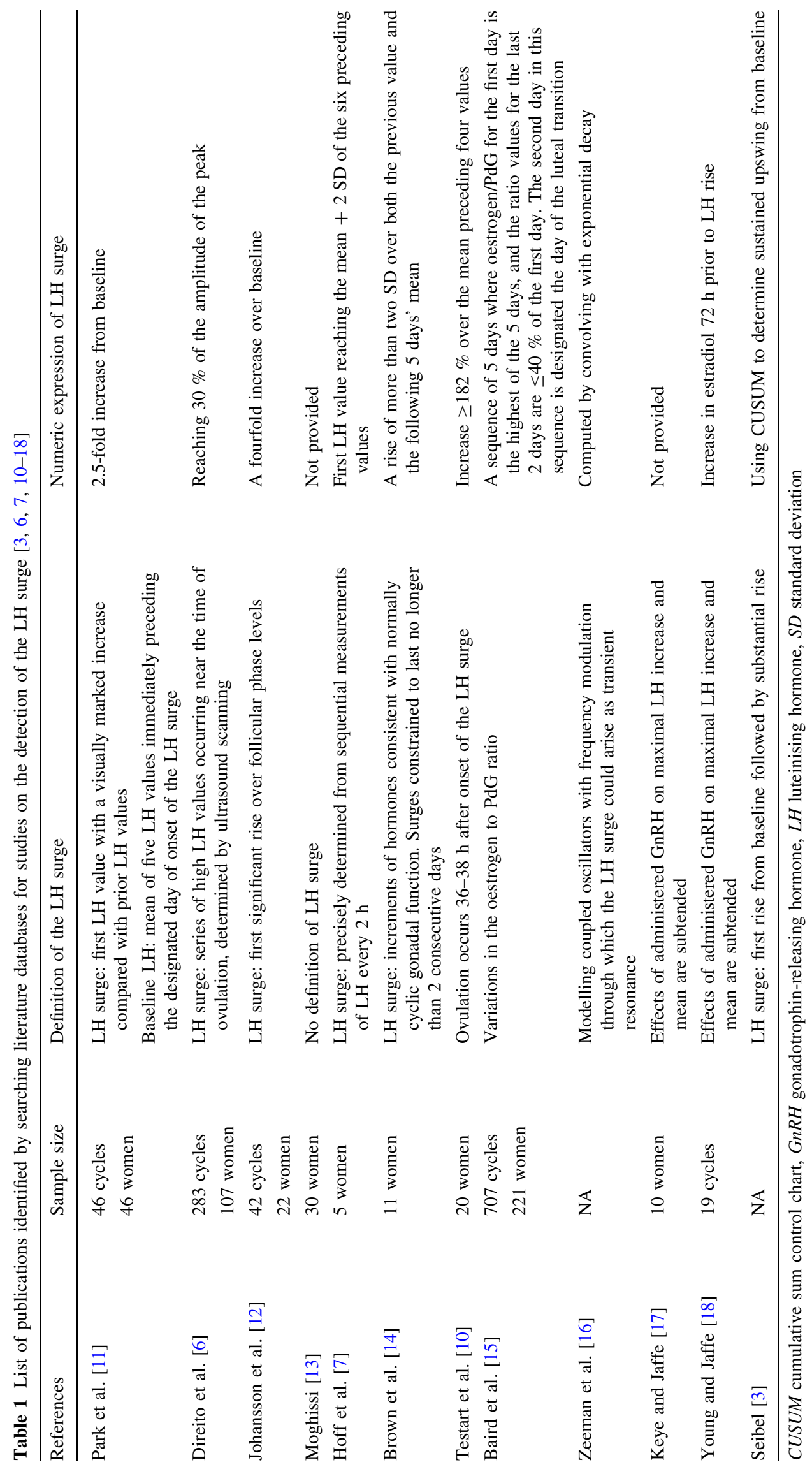


or 4 days. In total, eight variations of this method were observed; estimating the mean $\mathrm{LH}$ value of, either, the previous 3 or 4 days plus the reference day, which are days $3,4,5$ or 6 before the day of the LH peak. This method can only be applied retrospectively as data from the complete menstrual cycle are necessary for its application.

3. Using an estimate of the LH surge. This approach makes a rough estimation of the day of urinary LH surge by examination of the data of the cycle under investigation. It then takes days 1 or 2 before the estimated LH surge, as a reference level, plus the level on the previous 3 or 4 days, to calculate the mean value of LH. Four variants of this approach are documented. This method also can only be applied retrospectively as it requires data from the complete menstrual cycle.

\section{Comparison of the methods used to determine baseline value of $\mathrm{LH}$}

For all of the methods identified above, the baseline LH level was calculated. The method-dependent surge was then determined as the first sustained rise in LH above the baseline, with a sustained rise defined as a level of at least 2.5-fold of the SD over the mean baseline level. Figure 1 shows an example of complete hormonal profiles of $\mathrm{LH}$ during the menstrual cycle, highlighting the references used by each of the methods to determine the baseline value of LH in the follicular phase of the cycle.

\section{Performance of the identified methods}

for identifying the LH surge in complete urinary hormonal profiles of a reliable sample of menstrual cycles

Table 2 shows the results of the analysis evaluating the performance of all the different methods identified in the scientific literature for determining the onset of the $\mathrm{LH}$ surge in the 254 complete hormonal profiles analysed, whereby the surge day calculated by the various methods identified in the literature was compared with the reference surge day (as determined by an examination of the graphical cycle images by an expert panel).

\section{Method category 1 based on fixed days to estimate the baseline levels of $\mathrm{LH}$}

Four variants are reported in the literature that use fixed days during the cycle to estimate baseline urinary $\mathrm{LH}$ levels. These methods provided correct surge detection in 44.5-52.8\% of cycles (dependent on exact method). The values obtained for each specific method were: $44.5 \%$
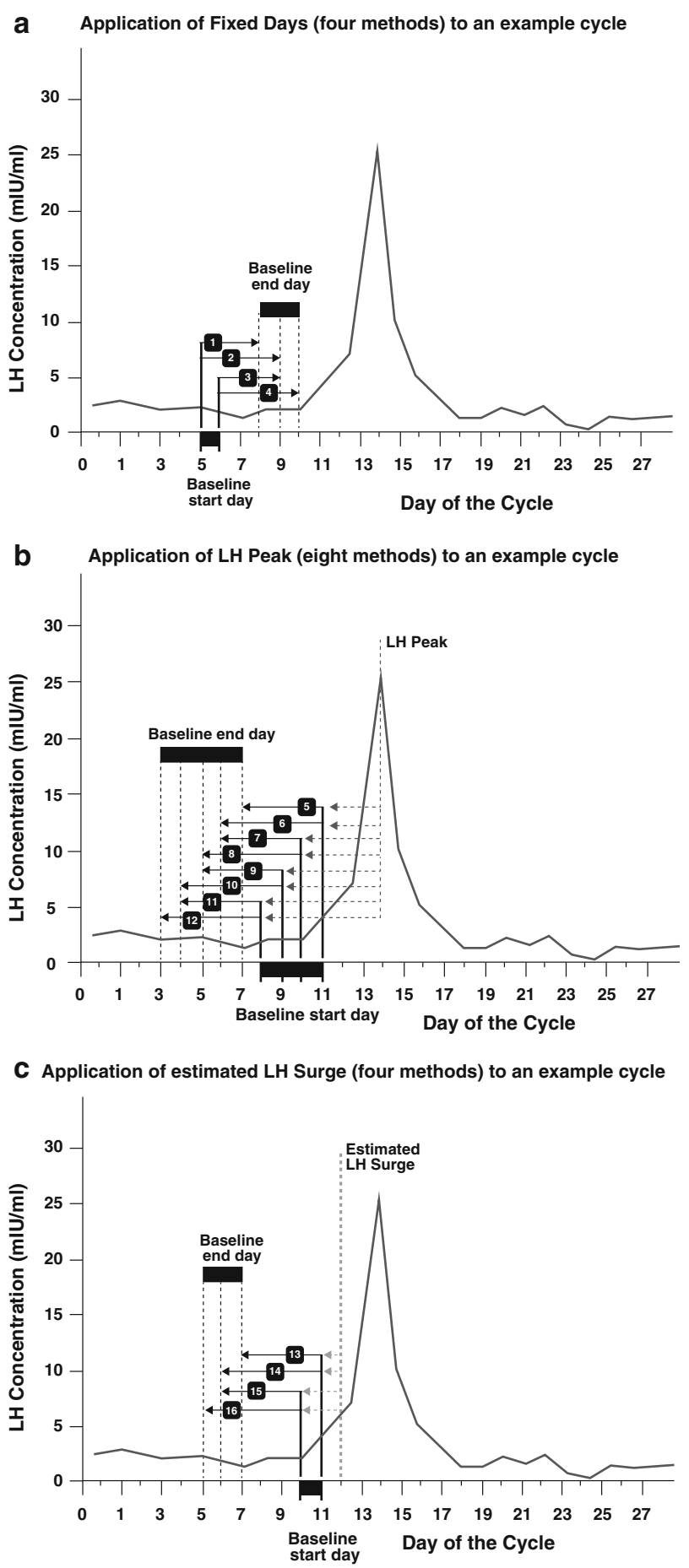

Fig. 1 Application of methods to determine baseline for LH surge identification to an example menstrual cycle. Each individual method is numbered: a using fixed days to calculate baseline, b using LH peak to calculate baseline, $\mathbf{c}$ using estimated $\mathrm{LH}$ surge to calculate baseline

when using cycle days 5-8; $48.4 \%$ when using days 5-9; $48.0 \%$ when using days 6-9; $52.8 \%$ when using days 6-10. The performance of these methods for identifying the surge on \pm 1 day of the reference surge ranged between 52.0 and $63.8 \%$. 


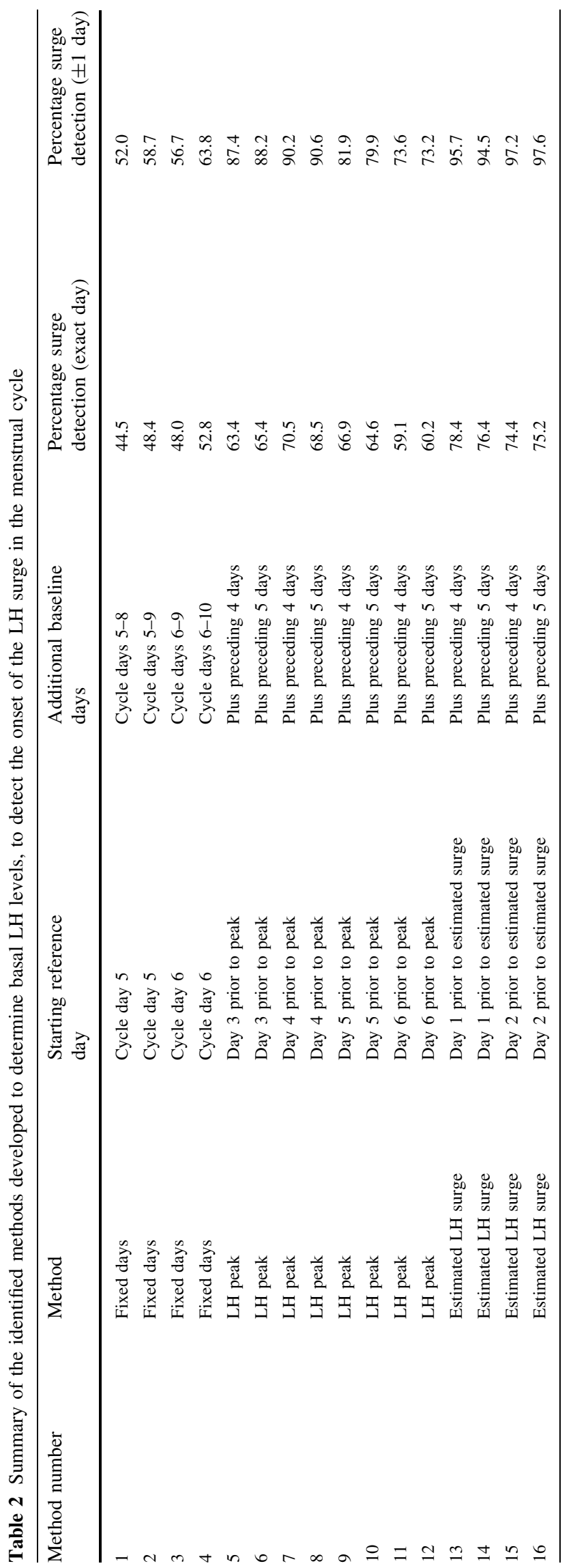

Method category 2 based on the day of the LH peak to estimate baseline LH levels

When the reference to determine the baseline values was the urinary LH peak, the approaches identified performed better than the fixed days method, detecting 63.4-70.5\% of the surges correctly, depending on which set of the eight variants of this category were applied.

When the reference was taken on day 3 before the $\mathrm{LH}$ peak, plus the previous 3 days to determine baseline values, the surge was correctly identified in $63.4 \%$ of the cases; however, when it was day 3 before the peak plus the previous 5 days, it was identified correctly in $65.4 \%$ of cases.

When the reference started on day 4 before the peak plus the previous 3 days, the surge was correctly identified in $70.5 \%$ of cases, and if taking the previous 4 days instead, it correctly identified $68.5 \%$ of the cases. When the reference was day 5 before the peak, plus the previous 3 days, it correctly identified the surge in $67 \%$ of the cases, whereas if it took the previous 4 days instead, it correctly identified $64.6 \%$ of the cases.

Finally, when the reference started on day 6 before the peak, plus the previous 3 days, it correctly identified the surge in $59.1 \%$ of the cases, whereas when taking the previous 4 days it correctly identified $60.2 \%$ of cases.

When considering the performance at determining the surge \pm 1 day of the reference surge, the range rose to $73.3-90.5 \%$.

\section{Method category 3 based on the day of the LH surge} to estimate baseline LH levels

Methods using the estimated urinary LH surge as a reference to determine the baseline $\mathrm{LH}$ value were observed to offer superior performance in correctly detecting the surge, finding them correctly ranged from 74.4 to $78.4 \%$.

When the reference to estimate baseline values started from day 1 before the estimated surge plus the previous 3 days, it correctly identified the surge in $78.4 \%$ of the cases, whereas taking the previous 4 days instead identified $76.4 \%$ of the surges correctly. When the reference used was day 2 before the estimated surge plus the previous 3 days, it correctly identified $74.4 \%$ of the cases, whereas using 4 days, produced $75.2 \%$ of correct identifications.

The performance of these methods at finding the surge within \pm 1 day of the reference surge ranged from 94.5 to $97.6 \%$.

\section{Discussion}

Prediction of ovulation can be important for women who are trying to conceive, in order for them to time intercourse to their fertile period to maximise their chances of 
conception. It can also have clinical importance for appropriate timing of procedures, such as intrauterine insemination, frozen embryo transfer or donor sperm insemination.

Over the years, several methods have been developed to predict ovulation. One of the most common methods used in fertility awareness is the sympto-thermal method (STM) because abundant evidence of its reliability has been presented [22-25]. However, the STM requires training in use, detailed recording of symptoms of self-observation and the application of several calculation rules, all lowering general acceptance rates. With the advancement of technology, other reliable, objective and less user-dependent methods have been introduced, which rely on the analysis of the levels of reproductive hormones measured in urine [26]. The study of hormonal profiles in urine during the menstrual cycle has proved to be very valuable in understanding menstrual cycle dynamics, as well as variability in ovulation [19, 27, 28]. Likewise, urinary hormone testing has become an established application for personal monitoring of fertility awareness [9]. Both temperature and hormonal analysis using repeated measurements in menstrual cycles are currently under intensive research as part of the evolving field of digital signal tracking in gynaecological endocrinology [21].

In premenopausal women, the menstrual cycle has a recognisable general pattern, and an average duration; however, there are important variations in cycle length and dynamics, both between and within women [29-33]. Likewise, there are differences in hormonal patterns, even in ovulatory cycles, which introduce a further source of difficulty into the analysis [34, 35]. This compromises the utility of all fertility awareness methods that solely rely on statistical parameters or calculation rules of the menstrual cycle (i.e. monitoring cycle days), and strengthens the importance of looking at specific events of physiological relevance leading to ovulation, such as the LH surge. It is the LH surge which always precedes ovulation, rather than the LH peak, and which is a signal of impending ovulation and peak fertility. Therefore, it is important to have a reliable algorithm to determine the onset of the LH surge in population studies. A standardised algorithm for determining the timing of the urinary LH surge in studies that have examined the hormone profiles in women is still not available.

This study examined the scientific literature, searching for studies focusing on the detection of the onset of the $\mathrm{LH}$ surge during the cycle, in urine samples, and found 12 relevant studies. In these papers, there were three major methodological categories designed and used to determine the onset of the surge. The key difference between these methods was the reference used for estimating the baseline values of urinary LH during the follicular phase. Important differences in the performance of these methods were identified. Methods that used the LH surge as a reference to estimate baseline values of LH clearly had the highest level of reliability in this respect, detecting on average up to $76.1 \%$ of the surges, compared with only $64.8 \%$ when the LH peak was used as a reference and $48.4 \%$ when using fixed days of the cycle. The findings of this study might help to improve the analysis of the human menstrual cycle for monitoring to assist with conception and contraception, as well as for biomedical research. In addition, a common methodology would facilitate comparisons between studies.

Many studies report LH peak [29, 36]; however, as a prospective measure, peak LH has less value as it often occurs post ovulation [37]. In addition, assignment of the day of peak urinary LH is dependent on the assay used [4]. Some urinary LH assays use antibodies that recognise the beta-core fragment of $\mathrm{LH}$ (LH- $\beta \mathrm{cf})$, which is a by-product of LH metabolism, and the predominant molecular form of $\mathrm{LH}$ in the urine [38]. Levels of LH- $\beta \mathrm{cf}$ continue to rise after the level of the physiologically relevant intact LH reaches peak concentration, sometimes achieving maximal levels up to 5 days later [4].

The comparatively poor performance of this prospectively applicable approach that uses fixed days during the menstrual cycle to determine baseline levels of LH is somewhat expected, since this approach does not take into consideration important factors, such as the variability in the length of the cycle, which mostly affects the follicular phase and exerts an influence over the hormonal dynamics [30]. Ovulation has been seen as early as day 8 of the menstrual cycle and, as the fixed days method generally includes day 8 , it is clear that this method will fail under such circumstances.

The higher reliability of a mid-cycle reference (method \#2) to determine baseline LH was anticipated, because data from the complete menstrual cycle have to be considered for its application, and due to its proximity with the fertile window, which is a very stable stage of the cycle, and unlike the early follicular phase, is not affected by age [39, 40]. It is also clear why the approach that uses the estimated surge (method \#3) as a reference performs better than the method using the peak (method \#2) because in almost $25 \%$ of normal menstrual cycles the peak may occur postovulatory (Roos et al. submitted); differences in the LH dynamics around the time of the surge and peak in LH account for this. Some complex LH peaks have been described when researchers have used an assay that recognises $\mathrm{LH}-\beta \mathrm{cf}$, with a longer duration than normal, sometimes described as 'biphasic' or in 'plateau' $[6,11]$; this often presents a significant delay between the surge and the peak of LH, compared with the 'spike' type of peaks. It is possible that when the reference is taken starting 3 or 4 days before the peak, in this type of cycle, 
levels of LH are already high, decreasing the accuracy of the estimation of the baseline levels. Likewise, when using a large number of days away from the peak (such as 6 or 5), in short cycles or those of normal duration, the levels estimated might be closer to those found in the fixed approach.

For fertility monitors, however, a retrospective approach with analysis of data from a complete menstrual cycle is not applicable. Therefore, learning monitors should consider the location reference points from previous cycles for higher reliability of surge detection. Current research focuses on a formal mathematical approach (signal tracking), finding distinct patterns in hormonal and temperature curves to predict change points and distinguish them from the random 'noise' (change-point analysis) [21].

A further way to improve the results includes other hormonal markers in urine, in addition to LH. These are estradiol and its metabolites, since a rise in the levels of estradiol triggers the rise in the levels of LH [38], and peak estrone-3-glucuronide (E3G) levels occur on the day of ovulation, as do those of follicle-stimulating hormone (FSH). However, this level of detail would add considerable complexity and additional cost to the analysis, especially by repeated measurement.

Ultrasound-observed ovulation is the gold-standard method used to determine the timing of ovulation and it would be of interest to observe how different approaches to LH surge definition compare to this reference. However, there is variability between women in the timing of the $\mathrm{LH}$ surge in relation to ultrasound-observed ovulation [6], thus further work could consider this relationship.

In conclusion, this study found that there are many methods for calculating the LH surge in urine reported in the literature, and their effectiveness at correctly identifying the surge varies between methods. Some of these methods are in use in current fertility monitors. Care should be taken when comparing between studies reporting urinary LH surge data, as results will be dependent on the method employed for surge identification. This study supports the recommendation for using methods that involve an initial estimation of the LH surge to establish which days should be used to determine baseline.

Acknowledgments This work was funded and conducted by employees of Swiss Precision Diagnostics (SPD) Development Company Ltd., a subsidiary of SPD GmbH, which manufactures and sells Clearblue pregnancy and fertility home tests. Some of the study results reported in this manuscript were previously presented at the 13th European Society of Contraception and Reproductive Health (ESC) Congress held in Lisbon in 2014.

Conflict of interest Sonya Godbert, Fernando Miro, Chris Shreeves and Sarah Johnson are employees (or previous employees) of SPD Development Company Ltd., a wholly owned subsidiary of SPD Swiss Precision Diagnostics $\mathrm{GmbH}$, the manufacturers of
Clearblue $^{\mathrm{TM}}$ pregnancy and fertility tests. Christian Gnoth has been a principal investigator of the MeMo study, partly supported by SPD Development Company Ltd. All authors have full control of all the primary data and agree to allow the journal review if requested.

Open Access This article is distributed under the terms of the Creative Commons Attribution 4.0 International License (http:// creativecommons.org/licenses/by/4.0/), which permits unrestricted use, distribution, and reproduction in any medium, provided you give appropriate credit to the original author(s) and the source, provide a link to the Creative Commons license, and indicate if changes were made.

\section{References}

1. Fritz MA, Speroff L (2011) How hormones work. In: Fritz MA, Speroff L (eds) Clinical gynecologic endocrinology and infertility, 8th edn. Lippincott Williams \& Wilkins, Pennsylvania

2. Stanford JB, White JL, Hatasaka H (2002) Timing intercourse to achieve pregnancy: current evidence. Obstet Gynecol 100:13331341

3. Seibel M (1986) Luteinizing hormone and ovulation timing. J Reprod Med 31:754-759

4. Johnson S, Weddell S, Godbert S, Freundl G, Roos J, Gnoth C (2015) Development of the first urinary reproductive hormone ranges referenced to independently determined ovulation day. Clin Chem Lab Med. doi:10.1515/cclm-2014-1087

5. Stricker R, Eberhart R, Chevailler M, Quinn F, Bischof P, Stricker R (2006) Establishment of detailed reference values for luteinizing hormone, follicle stimulating hormone, estradiol, and progesterone during different phases of the menstrual cycle on the Abbott ARCHITECT analyser. Clin Chem Lab Med 44:883887

6. Direito A, Bailly S, Mariani A, Ecochard R (2013) Relationships between the luteinizing hormone surge and other characteristics of the menstrual cycle in normally ovulating women. Fertil Steril 99:279-285

7. Hoff JD, Quigley ME, Yen SSC (1983) Hormonal dynamics at midcycle: a re-evaluation. J Clin Endocrinol Metab 57:792-796

8. Howards PP, Schisterman EF, Wactawski-Wende J, Reschke JE, Frazer AA, Hovey KM (2009) Timing clinic visits to phases of the menstrual cycle by using a fertility monitor: the BioCycle Study. Am J Epidemiol 169:105-112

9. Behre HM, Kuhlage J, Gaßner C, Sonntag B, Schem C, Schneider HPG, Nieschlag E (2000) Prediction of ovulation by urinary hormone measurements with the home use ClearPlan ${ }^{\circledR}$ Fertility Monitor: comparison with transvaginal ultrasound scans and serum hormone measurements. Hum Reprod 15:2478-2482

10. Testart J, Frydman R, Feinstein MC, Thebault A, Roger M, Scholler $\mathrm{R}$ (1981) Interpretation of plasma luteinizing hormone assay for the collection of mature oocytes from women: definition of a luteinizing hormone surge-initiating rise. Fertil Steril 36:50-54

11. Park SJ, Goldsmith LT, Skurnick JH, Wojtczuk A, Weiss G (2007) Characteristics of the urinary luteinizing hormone surge in young ovulatory women. Fertil Steril 88:684-690

12. Johansson EDB, Wide L, Gemzell C (1971) Luteinizing hormone (LH) and progesterone in plasma and $\mathrm{LH}$ and oestrogens in urine during 42 normal menstrual cycles. Acta Endocrinol 68:502-512

13. Moghissi KS (1976) Accuracy of basal body temperature for ovulation detection. Fertil Steril 27:1415-1420

14. Brown JR, Skurnick JH, Sharma N, Adel T, Santoro N (1993) Frequent intermittent ovarian function in women with premature menopause: a longitudinal study. Endocr J 1:467-474 
15. Baird DD, Weinberg CR, Wilcox AJ, McConnaughey DR (1991) Using the ratio of urinary oestrogen and progesterone metabolites to estimate day of ovulation. Stat Med 10:255-266

16. Zeeman ML, Weekesser W, Gokhman D (2003) Resonance in the menstrual cycle: a new model of the LH surge. Reprod Biomed Online 7:295-300

17. Keye WR, Jaffe RB (1975) Strength-duration characteristics of estrogen effects on gonadotropin response to gonadotropin-releasing hormone in women. I. Effects of varying duration of estradiol administration. J Clin Endocrinol Metab 41:1003-1008

18. Young JR, Jaffe RB (1976) Strength-duration characteristics of estrogen effects on gonadotropin response to gonadotropin-releasing hormone in women. II. Effects of varying concentrations of estradiol. J Clin Endocrinol Metab 42:432-442

19. Miro F, Parker SW, Aspinall LJ, Coley J, Perry PW, Ellis JE (2005) Sequential classification of endocrine stages during reproductive aging in women: the FREEDOM study. Menopause 12:281-290

20. Frank-Herrmann P, Heil J, Gnoth C, Toledo E, Baur S, Pyper C, Jenetzky E, Strowitzki T, Freundl G (2007) The effectiveness of a fertility awareness based method to avoid pregnancy in relation to a couple's sexual behaviour during the fertile time: a prospective longitudinal study. Hum Reprod 22:1310-1319

21. Freundl G, Frank-Herrmann P, Brown S, Blackwell L (2014) A new method to detect significant basal body temperature changes during a woman's menstrual cycle. Eur J Contracept Reprod Health Care 19:392-400

22. Guida M, Tommaselli GA, Palomba S, Pellicano M, Moccia G, Di Carlo C, Nappi C (1999) Efficacy of methods for determining ovulation in a natural family planning program. Fertil Steril 72:900-904

23. Frank-Herrmann P, Gnoth C, Baur S, Strowitzki T, Freundl G (2005) Determination of the fertile window: reproductive competence of women-European cycle database. Gynecol Endocrinol 20:305-312

24. Bigelow JL, Dunson DB, Stanford JB, Ecochard R, Gnoth C, Colombo B (2004) Mucus observations in the fertile window: a better predictor of conception than timing of intercourse. Hum Reprod 19:889-892

25. Gnoth C, Freundl G, Frank-Herrmann P (2002) Natural family planning and the management of infertility. Arch Gynecol Obstet 267:67-71

26. Fehring RJ, Schneider M, Raviele K, Rodriquez D, Pruszynski J (2013) Randomized comparison of two Internet-supported fertility-awareness-based methods of family planning. Contraception 88:24-30
27. Metcalf MG, Donald RA, Livesey JH (1981) Pituitary-ovarian function in normal women during the menopausal transition. Clin Endocrinol 14:245-255

28. Santoro N, Brown JR, Adel T, Skurnick JH (1986) Characterization of reproductive hormonal dynamics in the perimenopause. J Clin Endocrinol Metab 81:1495-1501

29. Cole LA, Ladner DG, Byrn FW (2009) The normal variabilities of the menstrual cycle. Fertil Steril 91:522-527

30. Johnson SR, Miro F, Barrett S, Ellis JE (2009) Levels of urinary human chorionic gonadotrophin (hCG) following conception and variability of menstrual cycle length in a cohort of women attempting to conceive. Curr Med Res Opin 25:741-748

31. Matsumoto S, Nogami Y, Ohkuri S (1962) Statistical studies on menstruation: a criticism on the definition of normal menstruation. Gunma J Med Sci 11:294-318

32. Latz LJ, Reiner E (1935) Natural conception control. JAMA 105:1241-1246

33. Creinin MD, Keverline S, Meyn LA (2004) How regular is regular? An analysis of menstrual cycle regularity. Contraception 70:289-292

34. Blackwell LF, Vigil P, Cooke DG, d'Arcangues C, Brown JB (2013) Monitoring of ovarian activity by daily measurement of urinary excretion rates of oestrone glucuronide and pregnanediol glucuronide using the Ovarian Monitor, Part III: variability of normal menstrual cycle profiles. Hum Reprod 28:3306-3315

35. Alliende ME (2002) Mean versus individual hormonal profiles in the menstrual cycle. Fertil Steril 78:90-95

36. Lenton EA, Landgren B, Sexton L (1984) Normal variation in the length of the luteal phase of the menstrual cycle: identification of the short luteal phase. Br J Obstet Gynecol 91:685-689

37. World Health Organization Task Force Investigators (1980) Temporal relationships between ovulation and defined changes in the concentration of plasma estradiol-17 $\beta$, luteinizing hormone, follicle stimulating hormone, and progesterone. Am J Obstet Gynecol 138:383-390

38. Wilcox AJ, Dunson D, Baird DD (2000) The timing of the "fertile window" in the menstrual cycle: day specific estimates from a prospective study. BMJ 321:1259-1262

39. Iles RK, Javid MK, Gunn LK, Chard T (1999) Cross-reaction with luteinizing hormone beta-core is responsible for the agedependent increase of immunoreactive beta-core fragment of human chorionic gonadotropin in women with nonmalignant conditions. Clin Chem 45:532-538

40. Dunson DB, Colombo B, Baird DD (2001) Changes with age in the level and duration of fertility in the menstrual cycle. Hum Reprod 17:1399-1403 